\title{
SENTIDO DE VIDA, BEM-ESTAR SUBJETIVO E BEM-ESTAR ESPIRITUAL EM JOVENS PORTUGUESES E BRASILEIROS
}

\author{
Maria da Luz Vale-Dias \\ Faculdade de Psicologia e de Ciências da Educação \\ Universidade de Coimbra, Portugal \\ valedias@fpce.uc.pt \\ Juliana Sant'Anna dos Santos Veras \\ Faculdade de Psicologia e de Ciências da Educação \\ Universidade de Coimbra, Portugal \\ julianassveras@gmail.com
}

Recepción Artículo: 31 enero 2020 Admisión Evaluación: 4 marzo 2020

Informe Evaluador 1: 1 enero 2020

Informe Evaluador 2: 1 enero 2020

Aprobación Publicación: 20 abril 2020

\section{RESUMO}

Introdução: Entre as dimensões para a felicidade do ser humano, assumem importância o sentido de vida (SV), bem-estar espiritual (BEE) e bem-estar subjetivo (BES). Neste estudo avalia-se a capacidade de prever 0 BEE e o BES a partir das dimensões do SV. Apresentam-se dados com alcance intercultural, envolvendo amostras do Brasil e de Portugal. Método: A amostra brasileira possui 300 sujeitos (71.3\% do sexo feminino). A amostra portuguesa inclui 298 sujeitos (64.1\% do sexo feminino). Nas duas amostras, a média de idade é de aproximadamente 20 anos. Na recolha de dados, recorremos a diversos questionários relativos aos construtos em análise, adaptados aos dois países. Resultados: Nas duas amostras, a dimensão presença de SV é preditora, apenas com uma exceção, dos fatores do BEE e do BES. Na amostra portuguesa, a procura de SV também se revela preditora de alguns destes fatores. A religião revelou-se relevante para a presença de SV, sendo esta significativamente mais elevada nos sujeitos que dizem ter uma religião. Observou-se que a religião influencia o SV e, em certa medida, o BEE. O SV e a dimensão transcendental do BEE foram significativamente mais elevados na amostra brasileira. Foram encontradas escassas diferenças em função do sexo e apenas na amostra brasileira. Discussão: Refletindo sobre os resultados mais relevantes e suas limitações, faz-se a proposta de novas investigações, salientando a importância do acompanhamento dos jovens nos três pilares em estudo.

Palavras-chave: sentido de vida; bem-estar subjetivo; bem-estar espiritual; jovens; adulto emergente

\section{ABSTRACT}

Meaning in life, subjective well-being and spiritual well-being in Portuguese and Brazilian young people. Introduction: Among the dimensions that determine the happiness of the human being, the 


\section{SENTIDO DE VIDA, BEM-ESTAR SUBJETIVO E BEM-ESTAR ESPIRITUAL EM JOVENS PORTUGUESES E BRASILEIROS}

meaning in life (ML), spiritual well-being (SpWB) and subjective well-being (SubjWB) are of particular interest. In this research, we evaluate the capacity to predict SpWB and SubjWB from the dimensions of the ML. We intend to present data with some intercultural reach, using samples from Brazil and Portugal. Method: The Brazilian sample has 300 subjects (71.3\% female). As for the Portuguese sample, it consists of 298 subjects (64.1\% female). In both samples, the mean age is approximately 20 years. For the data collection, we used several questionnaires adapted to the two countries. Results: In both samples, the presence of $\mathrm{ML}$ is predictor, with only one exception, of the factors of SpWB and SubjWB. In the Portuguese sample, the search for ML is also a predictor of some of these factors. Religion is relevant for the presence of $\mathrm{ML}$, being this one significantly higher in the group of subjects who say they have a religion. It was also observed that religion influences the ML and, to a certain extent, the SpWB. There were also significantly higher results in the $M L$ and in the transcendental dimension of SpWB in the Brazilian sample. Few differences were found according to sex, only in the Brazilian sample. Discussion: It is presented a reflection on the most relevant results, their limitations and proposals of new investigations, stressing the importance of accompanying young people in these three pillars.

Keywords: meaning in life; subjective well-being; spiritual well-being; young people; emerging adult

\section{INTRODUÇÃO}

0 ser humano encontra-se em constante desenvolvimento, sendo relevante considerar algumas dimensões determinantes para o seu funcionamento e bem-estar (Albuquerque, \& Tróccoli, 2004; Carvalho, \& Vale Dias, 2013; Diener, Lucas, \& Oshi, 2002). Entre elas, assumem um interesse particular o sentido de vida, bem-estar subjetivo e bem-estar espiritual.

0 bem-estar, amplamente estudado pela Psicologia Positiva, implica não só a referência a sentimentos felizes trazidos por algo agradável, mas também o sentimento de que o que se faz com a vida tem sentido e propósito. 0 primeiro aspeto, hedonista, refere-se ao bem-estar subjetivo (BES) e implica as avaliações cognitivas e afetivas acerca da vida (cf. Diener, Lucas \& Oshi, 2002), sendo que as suas componentes essenciais são a presença de emoções agradáveis, ausência de emoções negativas e satisfação com a vida. 0 segundo aspeto, eudemonista, relaciona-se com o facto de as pessoas compreenderem ou verem significado nas suas vidas, com o grau em que consideram ter um propósito, uma missão ou um objetivo abrangente na vida (Steger, Oishi, \& Kashdan, 2009).

0 sentido de vida (SV), profundamente explorado por Frankl (1979), pode entender-se como a medida em que as pessoas veem significado nas suas vidas, como a busca diária pelas certezas que guiam a vida e implica a própria capacidade de lutar pela felicidade. De acordo com Barros, "A busca de sentido abrange uma dimensão cognitivo-afectivo-motivacional, podendo considerar-se uma atitude existencial, meta do processo de crescimento espiritual do homem", sendo que "Só buscando (e encontrando) um significado duradouro para a vida, a pessoa humana pode ser minimamente feliz." (2009, 94). Vários autores consideram o SV como uma parte vital dos processos mentais e do comportamento (e.g., Frankl 1979; Steger 2012). É ainda considerado um elemento crucial no coping religioso e bem-estar psicológico, sendo usado para lidar com as dificuldades e desafios da vida (Krok, 2015). A evidência da relação entre bem-estar e SV é defendida por diversos autores, sendo que "0 sentido de vida mostrou estar positivamente associado ao bem-estar subjectivo (Steger e Frezien, 2005; Zika e Chamberlain, 1992) e à resistência ao stress (Moomal, 1999), e negativamente correlacionado com a ansiedade e depressão (Beken, 1997; Zika e Chamberlain, 1992), com o neuroticismo (Person e Sheffield, 1974) e com 0 abuso do álcool e outras substâncias (Debats, 1999)" (Simões et al., 2010, p.248). Atualmente, apesar de existirem diferentes definições de SV (e.g., Frankl, 1965; Baumeister, 1991; Emmons, 2003), há um grande consenso quanto à sua relevância, sendo considerado "um factor de primeira importância do bem-estar subjetivo" (Simões et al., 2010, p.247).

De acordo com Steger e col. (Steger, 2012; Steger, Oishi, \& Kesebir, 2011), a compreensão do SV remete para duas dimensões principais: a presença e a busca de SV. Ao efetuar um estudo ao longo da vida, o autor verificou que os indivíduos de estádios mais avançados referiam uma maior presença de sentido nas suas vidas, enquanto os que pertenciam a estádios mais precoces, como os adultos emergentes, reportavam níveis mais ele- 
vados de busca de SV (Steger, Oishi, \& Kashdan, 2009). Se considerarmos que a adultez emergente (Arnett, 2000) se caracteriza essencialmente por ser um período de grande exploração da identidade, em diferentes áreas, fará sentido este resultado. De qualquer forma, mesmo que os níveis de presença de SV variem ao longo do ciclo vital, a literatura considera que 0 sentimento de que a própria vida tem sentido é importante em qualquer etapa de desenvolvimento (Wong, 2000). Desta forma, segundo alguns autores (Steger, Oishi, \& Kashdan, 2009), espera-se uma relação positiva entre a presença de SV e diversos índices de bem-estar em qualquer etapa da vida.

Pela sua relevância na área da saúde, o presente estudo destaca ainda a dimensão espiritual do bem-estar. Fisher (1999) apresenta um modelo conceptual que define o bem-estar espiritual (BEE) como uma forma de estar dinâmica, que se manifesta na qualidade das relações estabelecidas em quatro domínios da existência humana: consigo próprio (domínio pessoal), com os outros (domínio comunitário), com o ambiente (domínio ambiental) e com algo ou Alguém que transcende o domínio humano (domínio transcendental). Uma vez que "o bem-estar espiritual é entendido como uma sensação de bem-estar que é experimentado quando encontramos um propósito que justifica o nosso comprometimento com algo da vida, e esse propósito envolve um significado último para a vida" (Marques, Sarriera e Dell Aglio, 2009, p.180), será expectável que se relacione com o próprio SV.

\section{OBJETIVOS DA INVESTIGAÇÃO}

Nesta investigação, com adultos emergentes (Arnett, 2000), procura-se, em primeiro lugar, compreender se 0 sentido de vida leva ao bem-estar. Assim, pretende-se avaliar a capacidade de prever 0 BEE e 0 BES a partir das dimensões do SV.

Num outro momento, procura-se saber se a religião influencia os construtos principais em análise. Será também analisado 0 efeito do sexo dos sujeitos.

Finalmente, pretende-se apresentar dados com algum alcance intercultural, envolvendo amostras de dois países.

\section{AMOSTRA}

0 presente estudo envolve amostras, não probabilísticas e ocasionais, de dois países diferentes, isto é, Brasil e Portugal. A amostra brasileira possui 300 indivíduos, 86 do sexo masculino (28.7\%) e 214 do sexo feminino (71.3\%). A amostra portuguesa (n=298) é constituída por 107 sujeitos do sexo masculino (35.9\%) e 191 do sexo feminino (64.1\%). Em ambas as amostras, de estudantes universitários, a média de idade é de aproximadamente 20 anos (Brasil: $M=20.23 ; D P=2.27$; Port.: $M=20.06 ; D P=2.07$ ).

\section{METODOLOGIA E INSTRUMENTOS}

Para a recolha de dados, utilizaram-se diversos questionários, que foram preenchidos individualmente pelos sujeitos, após o seu consentimento informado. Recorreu-se aos seguintes instrumentos:

1) Para a análise do SV foi usado o MLQ (Meaning in Life Questionnaire) na versão portuguesa (Simões et al., 2010) e na versão brasileira (QSV- Questionário de Sentido de Vida; Damásio et Koller, 2015);

2) Para medir o BEE, utilizou-Se o SWBQ (Spiritual Well-being Questionnaire), nas versões portuguesa (SWBQp; Gouveia, Marques, \& Ribeiro, 2009) e brasileira (QBEE - Questionário de Bem-Estar Espiritual; Dohms, Davoglio, \& Lettnin, 2015);

3) Quanto ao BES, recorreu-se à EBES (Escala de Bem-estar Subjetivo; Albuquerque \& Trocoli, 2004) para avaliar esta variável na amostra brasileira e à PANAS (Positive and Negative Affect Schedule; versão port.: Galinha, \& Pais-Ribeiro, 2005).) e à SWLS (Satisfaction With Life Scale; versão port.: Simões, 1992), na amostra portuguesa. estudo.

4) Nas duas amostras foram ainda aplicados questionários sociodemográficos, construídos para o presente

Os instrumentos apresentaram boas características psicométricas. Os valores de alfa de Cronbach são altos, com apenas dois valores abaixo de .80, especificamente na dimensão comunitária do BEE, quer na amostra portuguesa (.77) quer na brasileira (.73). 


\section{SENTIDO DE VIDA, BEM-ESTAR SUBJETIVO E BEM-ESTAR ESPIRITUAL EM}

JOVENS PORTUGUESES E BRASILEIROS

Na análise de dados, foram calculadas ANOVAs e regressões múltiplas. Os cálculos foram efetuados com 0 pacote estatístico IBM SPSS 22.0.

\section{RESULTADOS}

\section{Análise do efeito preditor do Sentido de Vida em relação ao Bem-Estar Espiritual e Bem-Estar} Subjetivo

Foram efetuadas regressões lineares, considerando ambas as amostras, tendo as duas subescalas do SV (procura e presença) como preditoras e as subescalas do BEE e BES como variáveis critério.

\section{Amostra Brasileira}

Tabela 1. Regressão linear do sentido de vida (QSV) tendo como variáveis critério o bem-estar espiritual (QBEE) e o bem-estar subjetivo (EBES) (amostra brasileira)

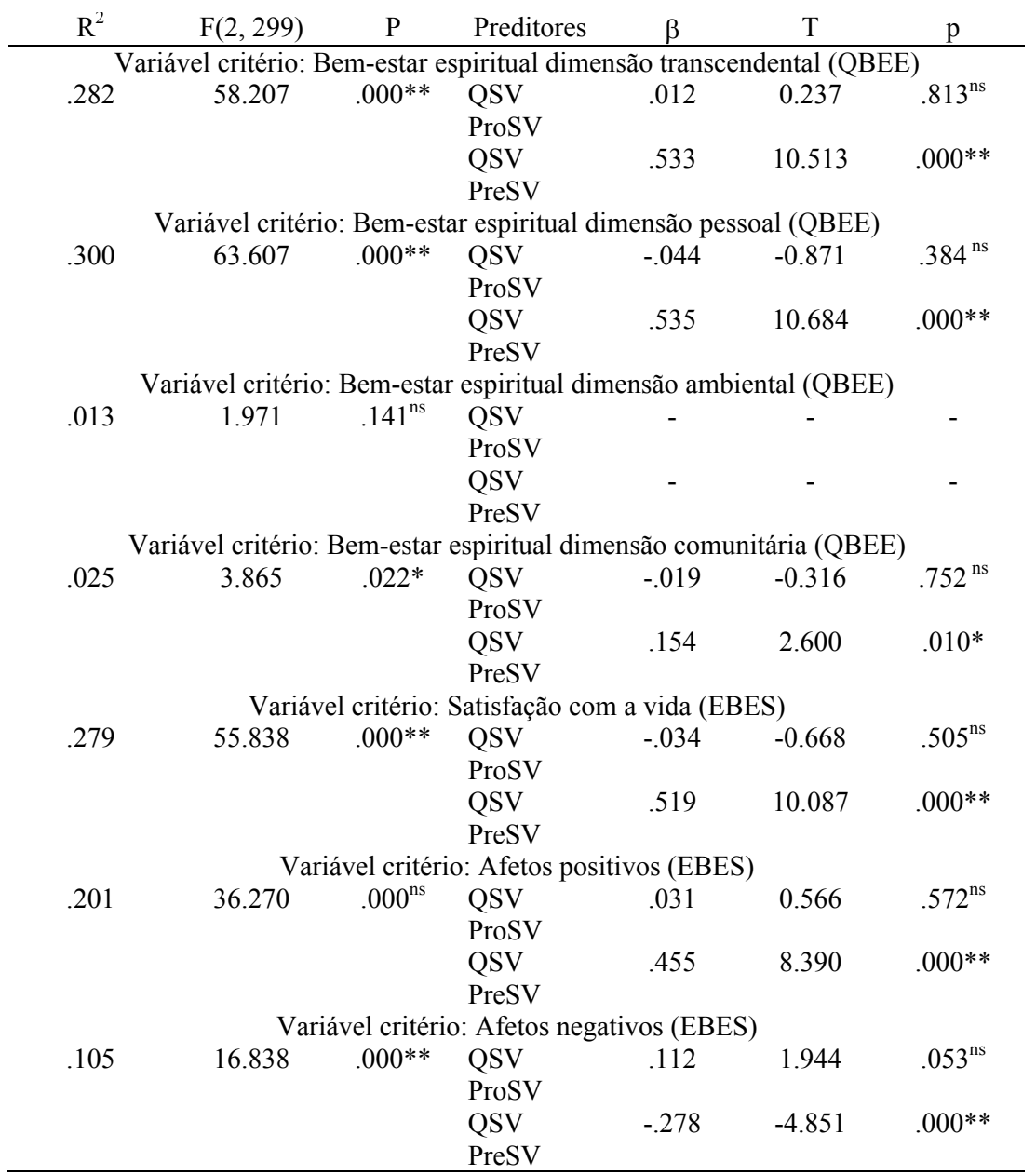

$* * p<.01 ; * * p<.05 ;{ }^{\text {ns }}$ não significativo. 
Quanto ao bem-estar espiritual (QBEE), nas dimensões transcendental $[F(2,299)=58.207, p<.001 ; \quad \beta$ PreSC $=.533, p<.001]$ e dimensão pessoal $[F(2,299)=63.607, p<.001 ; \beta$ PreSC $=.535, p<.001]$, apenas foi preditora a subescala presença de SV (QSV PreSV), com variâncias explicadas de $28.2 \%$ e $30 \%$, respetivamente. Na dimensão comunitária $[\mathrm{F}(2,299)=3.865, p=.022 ; \beta$ PreSC=.154, $p=.010]$, a presença de SV obteve significado estatístico mas a equação de regressão explica apenas $2.5 \%$ de variância. Nenhuma das variáveis do SV foi preditora da dimensão ambiental (Tabela 1).

A presença do SV também foi preditora da satisfação com a vida $[\mathrm{F}(2,299)=55.838, p<.001 ; \beta$ PreSC=.519, $p<.001]$, afetos positivos $[\mathrm{F}(2,299)=36.270, p<.001 ; \beta$ PreSC $=.455, p<.001] \mathrm{e}, \mathrm{com}$ sinal negativo, afetos negativos $[F(2,299)=16.838, p<.001 ; \beta$ PreSC $=-278, p<.001]$, explicando respetivamente $27.9 \%, 20.1 \%$ e $10.5 \%$ da variância.

\section{Amostra Portuguesa}

Como se verifica na Tabela 2, apesar de no seu conjunto a procura (MLQ ProSV) e a presença (MLQ PreSV) de SV explicarem apenas 11.4\% de variância, são ambas preditores significativos do BEE (SWBQp) na dimensão transcendental $\left[\mathrm{F}(2,294)=18.804, p<.001, \beta\right.$ ProSC $\left.=.129, p=.020 ; b_{\text {PreSC }}=.311 p<.001\right]$. Na dimensão pessoal apenas a presença de SV foi significativa e a equação de regressão explica $31.8 \%$ da variância $[F(2,294)=$ $67.925, p<.001, \beta$ PreSC $=.563, p<.001]$. Nenhuma das variáveis do SV foi preditora da dimensão ambiental. Também na dimensão comunitária, a presença de SV foi significativa, porém a equação de regressão explica apenas $7 \%$ de variância $[\mathrm{F}(2,294)=10.937, p<.001, \beta$ PreSC $=.263, p<.001]$.

A presença de SV foi preditora da subescala afetos positivos, explicando $22.5 \%$ da variância $[F(2,294)=$ 41.393, $p<.001, \beta$ PreSC $=.471, p<.001]$.

Quanto à satisfação com a vida, ambas as subescalas do SV foram preditoras (11.7\% de variância explicada), porém a procura com sinal negativo $[\mathrm{F}(2,294)=19.288, p<.001, \beta$ ProSC $=-.124, p=.024 ; \beta$ PreSC $=.320, p<.001]$.

Na subescala afetos negativos as variáveis do SV explicam $14.9 \%$ da variância, tendo a presença de SV sinal negativo $[\mathrm{F}(2,294)=25.284, p<.001, \beta$ ProSC $=.182, p=.001 ; \beta$ PreSC $=-.342, p<.001]$. 
Tabela 2. Regressão linear do sentido de vida (MLQ) tendo como variáveis critério o bem-estar espiritual (SWBQp) e o bem-estar subjetivo (SWLS e PANAS) (amostra portuguesa)

\begin{tabular}{|c|c|c|c|c|c|c|}
\hline $\mathrm{R}^{2}$ & $\mathrm{~F}(2,294)$ & $\mathrm{P}$ & Preditores & $\beta$ & $\mathrm{t}$ & $\mathrm{P}$ \\
\hline \multicolumn{7}{|c|}{ Variável critério: Bem estar espiritual dimensão transcendental (SWBQp) } \\
\hline \multirow[t]{3}{*}{.114} & 18.804 & $.000 * *$ & $\begin{array}{l}\text { MLQ } \\
\text { ProSV }\end{array}$ & .129 & 2.338 & $.020^{*}$ \\
\hline & & & $\begin{array}{l}\text { MLQ } \\
\text { PreSV }\end{array}$ & .311 & 5.639 & $.000 * *$ \\
\hline & ável critér & Bem esta & espiritual di & nsão pe & 1 (SWBC & \\
\hline \multirow[t]{3}{*}{.318} & 67.925 & $.000 * *$ & $\begin{array}{l}\text { MLQ } \\
\text { ProSV }\end{array}$ & .019 & 0.388 & $.698^{\mathrm{ns}}$ \\
\hline & & & $\begin{array}{l}\text { MLQ } \\
\text { PreSV }\end{array}$ & .563 & 11.643 & $.000 * *$ \\
\hline & vel critéric & em estar & spiritual din & xão aml & $\mathrm{al}(\mathrm{SWB}$ & \\
\hline \multirow[t]{2}{*}{.017} & 2.570 & $.078^{\mathrm{ns}}$ & $\begin{array}{l}\text { MLQ } \\
\text { ProSV }\end{array}$ & - & - & - \\
\hline & & & $\begin{array}{l}\text { MLQ } \\
\text { PreSV }\end{array}$ & - & - & - \\
\hline \multicolumn{7}{|c|}{ Variável critério: Bem estar espiritual dimensão comunitária (SWBQp) } \\
\hline \multirow[t]{2}{*}{.070} & 10.937 & $.000 * *$ & $\begin{array}{l}\text { MLQ } \\
\text { ProSV }\end{array}$ & .022 & 0.384 & $.701^{\mathrm{ns}}$ \\
\hline & Variá & critério: & $\begin{array}{c}\text { MLQ } \\
\text { PreSV } \\
\text { atisfação co }\end{array}$ & $\begin{array}{l}.263 \\
\text { vida }(\end{array}$ & $\begin{array}{l}4.659 \\
\text { S) }\end{array}$ & $.000 * *$ \\
\hline \multirow[t]{2}{*}{.117} & 19.288 & $.000 * *$ & $\begin{array}{l}\text { MLQ } \\
\text { ProSV }\end{array}$ & -.124 & -2.261 & $.024 *$ \\
\hline & Var & el critéric & $\begin{array}{c}\text { MLQ } \\
\text { PreSV } \\
\text { Afetos posi }\end{array}$ & $\begin{array}{l}.320 \\
\text { (PAI }\end{array}$ & 5.821 & $.000 * *$ \\
\hline \multirow[t]{2}{*}{.225} & 41.393 & $.000 * *$ & $\begin{array}{l}\text { MLQ } \\
\text { ProSV }\end{array}$ & -.061 & -1.177 & $.240^{\mathrm{ns}}$ \\
\hline & Var & l critéric & $\begin{array}{c}\text { MLQ } \\
\text { PreSV } \\
\text { Afetos nega }\end{array}$ & $\begin{array}{l}.471 \\
\text { os (PAI }\end{array}$ & 9.096 & $.000^{* *}$ \\
\hline \multirow[t]{2}{*}{.149} & 25.284 & $.000 * *$ & $\begin{array}{l}\text { MLQ } \\
\text { ProSV }\end{array}$ & .182 & 3.354 & $.001 * *$ \\
\hline & & & $\begin{array}{l}\text { MLQ } \\
\text { PreSV }\end{array}$ & -.342 & -6.310 & $.000^{* *}$ \\
\hline
\end{tabular}

$* * p<.01 ; * * p<.05 ;{ }^{\text {ns }}$ não significativo. 


\section{Comparação das amostras brasileira e portuguesa quanto ao sentido de vida e ao bem-estar espiritual}

Não foi possível comparar as duas amostras quanto ao BES, devido à inexistência de versões similares, com confiabilidade, do mesmo instrumento nos dois países. Assim, procedeu-se à comparação dos sujeitos brasileiros e portugueses em termos do seu SV e BEE (Tabela 3).

A amostra brasileira obteve uma média significativamente superior na subescala procura do SV $(M=24.19$; $D P=106.49)$ relativamente à amostra portuguesa $(M=16.49 ; D P=4.50)$, sendo a diferença estatisticamente significativa $[F(1,488.435)=229.397, p<.001]$. Também na presença de SV os brasileiros obtiveram pontuações superiores ( $M=22.85 ; D P=7.27)$ aos portugueses ( $M=18.24$; $D P 4.39)$, sendo que a diferença teve significado estatístico $[F(1,491.918)=88.315, p<.001]$.

0 mesmo sentido de resultados foi encontrado na dimensão transcendental do BEE, com os brasileiros a apresentarem uma média de $3.45(D P=1.03)$ e os portugueses a obterem uma média de 2.36 ( $D P=2.36)$; a diferença foi estatisticamente significativa $[\mathrm{F}(1,593.297)=152.565, p<.001]$.

Tabela 3. Médias, desvios-padrão e ANOVA das escalas do sentido de vida e do bem-estar espiritual em função da nacionalidade

\begin{tabular}{|c|c|c|c|c|c|c|}
\hline & Nacionalidade & $n$ & $M$ & $D P$ & $F$ & $P$ \\
\hline \multirow{2}{*}{$\begin{array}{l}\text { Procura do Sentido } \\
\text { de Vida }\end{array}$} & Portuguesa & 298 & 16.49 & 4.50 & \multirow[t]{2}{*}{$229.397^{\mathrm{a}}$} & \multirow[t]{2}{*}{$.000 * *$} \\
\hline & Brasileira & 300 & 24.19 & 7.55 & & \\
\hline \multirow{2}{*}{$\begin{array}{l}\text { Presença do Sentido } \\
\text { de Vida }\end{array}$} & Portuguesa & 298 & 18.24 & 4.39 & \multirow[t]{2}{*}{$88.315^{\mathrm{a}}$} & \multirow[t]{2}{*}{$.000 * *$} \\
\hline & Brasileira & 300 & 22.85 & 7.27 & & \\
\hline \multirow{4}{*}{$\begin{array}{l}\text { Dimensão } \\
\text { transcendental } \\
\text { (Bem-Estar } \\
\text { Espiritual) } \\
\text { Dimensão pessoal } \\
\text { (Bem-Estar } \\
\text { Espiritual) }\end{array}$} & Portuguesa & 296 & 2.36 & 1.12 & \multirow[t]{2}{*}{$152.565^{\mathrm{a}}$} & \multirow[t]{2}{*}{$.000 * *$} \\
\hline & Brasileira & 300 & 3.45 & 1.03 & & \\
\hline & Portuguesa & 296 & 3.56 & 0.72 & 2.228 & $.136^{\mathrm{ns}}$ \\
\hline & Brasileira & 300 & 3.65 & 0.76 & & \\
\hline \multirow{2}{*}{$\begin{array}{l}\text { Dimensão } \\
\text { ambiental (Bem- } \\
\text { Estar Espiritual) }\end{array}$} & Portuguesa & 296 & 3.60 & 0.88 & \multirow[t]{2}{*}{0.717} & \multirow[t]{2}{*}{$.397^{\mathrm{ns}}$} \\
\hline & Brasileira & 300 & 3.54 & 0.83 & & \\
\hline \multirow{2}{*}{$\begin{array}{l}\text { Dimensão } \\
\text { comunitaria (Bem- } \\
\text { Estar Espiritual) }\end{array}$} & Portuguesa & 296 & 3.91 & 0.60 & \multirow[t]{2}{*}{2.881} & \multirow[t]{2}{*}{$.090^{\mathrm{ns}}$} \\
\hline & Brasileira & 300 & 3.99 & 0.56 & & \\
\hline
\end{tabular}

${ }^{* *} p<.01 ;{ }^{\text {ns }}$ não significativo; ${ }^{\mathrm{a}} \mathrm{O}$ teste de Levene foi significativo, tendo sido considerada a correção de Brown-Forsythe.

\section{Análise das escalas em função do sexo}

Testaram-se, através da análise de variância, ANOVA, as diferenças entre sexos nas várias escalas. Na amostra brasileira, foram encontradas diferenças estatisticamente significativas na EBES afetos positivos, com os sujeitos do sexo masculino $(M=3.42, D P=0.65)$ a obterem uma média significativamente superior aos sujeitos do sexo feminino $(M=3.21, D P=0.72), F(1,289)=5.636, p=.018$. As diferenças não foram estatisticamente significativas nas restantes escalas. 
Na amostra portuguesa, não foram encontradas diferenças significativas entre sexos em nenhuma das escalas.

\section{Comparação dos sujeitos religiosos e não religiosos nas variáveis em estudo} Amostra brasileira

Tabela 4. Médias, desvios-padrão e ANOVA das escalas em função da religião (amostra brasileira)

\begin{tabular}{|c|c|c|c|c|c|c|}
\hline & Religioso & $N$ & $M$ & $D P$ & $F$ & $P$ \\
\hline \multirow{2}{*}{$\begin{array}{l}\text { EBES } \\
\text { Afetos positivos }\end{array}$} & Sim & 210 & 3.29 & 0.70 & 0.843 & $.359^{\mathrm{ns}}$ \\
\hline & Não & 80 & 3.21 & 0.73 & & \\
\hline \multirow{2}{*}{$\begin{array}{l}\text { EBES } \\
\text { Afetos negativos }\end{array}$} & Sim & 210 & 2.37 & 0.73 & 0.854 & $.356^{\mathrm{ns}}$ \\
\hline & Não & 80 & 2.46 & 0.78 & & \\
\hline \multirow{2}{*}{$\begin{array}{l}\text { EBES } \\
\text { Satisfação com a } \\
\text { vida }\end{array}$} & Sim & 210 & 3.64 & 0.69 & 1.632 & $.202^{\mathrm{ns}}$ \\
\hline & Não & 80 & 3.52 & 0.68 & & \\
\hline \multirow{2}{*}{$\begin{array}{l}\text { QSV } \\
\text { Procura do } \\
\text { sentido de vida }\end{array}$} & Sim & 217 & 23.92 & 7.58 & 1.023 & $.313^{\mathrm{ns}}$ \\
\hline & Não & 82 & 24.91 & 7.51 & & \\
\hline \multirow{2}{*}{$\begin{array}{l}\text { QSV } \\
\text { Presença do } \\
\text { sentido de vida }\end{array}$} & Sim & 217 & 24.26 & 6.92 & $34.260^{\mathrm{a}}$ & $.000 * *$ \\
\hline & Não & 82 & 19.05 & 6.85 & & \\
\hline \multirow{2}{*}{$\begin{array}{l}\text { QBEE } \\
\text { Dimensão } \\
\text { transcendental }\end{array}$} & Sim & 217 & 3.79 & 0.79 & 92.391 & $.000 * *$ \\
\hline & Não & 82 & 2.54 & 1.06 & & \\
\hline \multirow{2}{*}{$\begin{array}{l}\text { QBEE } \\
\text { Dimensão pessoal }\end{array}$} & Sim & 217 & 3.69 & 0.73 & 2.518 & $.114^{\mathrm{ns}}$ \\
\hline & Não & 82 & 3.53 & 0.83 & & \\
\hline \multirow{4}{*}{$\begin{array}{l}\text { QBEE } \\
\text { Dimensão } \\
\text { ambiental } \\
\text { QBEE } \\
\text { Dimensão } \\
\text { comunitária } \\
\end{array}$} & Sim & 217 & 3.51 & 0.78 & $1.133^{\mathrm{a}}$ & $.288^{\mathrm{ns}}$ \\
\hline & Não & 82 & 3.62 & 0.94 & & \\
\hline & Sim & 217 & 3.99 & 0.54 & 0.013 & $.911^{\mathrm{ns}}$ \\
\hline & Não & 82 & 4.00 & 0.61 & & \\
\hline
\end{tabular}

${ }^{* *} p<.01 ;{ }^{\text {ns }}$ não significativo; ${ }^{\mathrm{a}} \mathrm{O}$ teste de Levene foi significativo, foi considerada a correção de Brown-Forsythe.

Na escala QSV presença de $S V$, os sujeitos que têm uma religião $(M=24.26, D P=6.92)$ apresentaram uma média superior àqueles que assinalaram não ter religião $(M=19.05, D P=6.85)$, sendo que a diferença foi estatisticamente significativa $[F(1,147.202)=34.260, p<.001]$ (Tabela 4).

Na dimensão transcendental do QBEE também foi observada uma diferença estatisticamente significativa $[F(1,297)=92.391, p<.001]$, com os que têm religião a ter uma média de $3.79(D P=0.79)$ e os que não têm com uma média de 2.54 ( $D P=1.06)$. 
Para as restantes escalas, as diferenças entre médias não foram estatisticamente significativas. Amostra portuguesa

Tabela 5. Médias, desvios-padrão e ANOVA das escalas em função da religião (amostra portuguesa)

\begin{tabular}{|c|c|c|c|c|c|c|}
\hline & Religião & $n$ & $M$ & $D P$ & $F$ & $P$ \\
\hline \multirow{2}{*}{$\begin{array}{l}\text { MLQ } \\
\text { Procura do sentido } \\
\text { de vida }\end{array}$} & Sim & 163 & 17.30 & 4.24 & 11.313 & $.001 * *$ \\
\hline & Não & 132 & 15.55 & 4.67 & & \\
\hline \multirow{2}{*}{$\begin{array}{l}\text { MLQ } \\
\text { Presença do sentido } \\
\text { de vida }\end{array}$} & Sim & 163 & 19.08 & 4.34 & 12.453 & $.000 * *$ \\
\hline & Não & 132 & 17.30 & 4.26 & & \\
\hline \multirow{2}{*}{$\begin{array}{l}\text { SWBQp } \\
\text { Dimensão } \\
\text { transcendental }\end{array}$} & Sim & 163 & 2.86 & 1.00 & 93.933 & $.000 * *$ \\
\hline & Não & 130 & 1.74 & 0.95 & & \\
\hline \multirow{2}{*}{$\begin{array}{l}\text { SWBQp } \\
\text { Dimensão pessoal }\end{array}$} & Sim & 163 & 3.63 & 0.70 & 2.448 & $.119^{\mathrm{ns}}$ \\
\hline & Não & 130 & 3.49 & 0.72 & & \\
\hline \multirow{2}{*}{$\begin{array}{l}\text { SWBQp } \\
\text { Dimensão ambiental }\end{array}$} & Sim & 163 & 3.64 & 0.84 & 0.447 & $.504^{\mathrm{ns}}$ \\
\hline & Não & 130 & 3.57 & 0.92 & & \\
\hline \multirow{4}{*}{$\begin{array}{l}\text { SWBQp } \\
\text { Dimensão } \\
\text { comunitária } \\
\text { PANAS } \\
\text { Afetos positivos }\end{array}$} & Sim & 163 & 3.98 & 0.59 & 4.932 & $.027 *$ \\
\hline & Não & 130 & 3.82 & 0.60 & & \\
\hline & Sim & 158 & 3.19 & 0.69 & .831 & $.363^{\mathrm{ns}}$ \\
\hline & Não & 133 & 3.11 & 0.65 & & \\
\hline \multirow{2}{*}{$\begin{array}{l}\text { PANAS } \\
\text { Afetos negativos }\end{array}$} & Sim & 158 & 2.15 & 0.75 & .485 & $.487^{\mathrm{ns}}$ \\
\hline & Não & 133 & 2.08 & 0.74 & & \\
\hline \multirow[t]{2}{*}{ SWLS } & Sim & 162 & 3.71 & 0.85 & 1.697 & $.194^{\mathrm{ns}}$ \\
\hline & Não & 132 & 3.58 & 0.83 & & \\
\hline
\end{tabular}

${ }^{*} p<.05 ; * * p<.01 ;{ }^{\mathrm{ns}}$ não significativo.

Os sujeitos que afirmaram ter uma religião obtiveram médias superiores na procura de SV ( $M=17.30$, $D P=4.24)$ relativamente àqueles que assinalaram não ter $(M=15.55, D P=4.67)$ e a diferença foi estatisticamente significativa [F(1,294)=11.313, $p=.001]$ (Tabela 5).

Também na subescala presença de SV, os que têm religião obtiveram uma média superior ( $M=19.08$; $D P=4.34)$ face aos que não têm $(M=17.30, D P=4.26)$, a diferença observada teve significado estatístico $[\mathrm{F}(1,294)=12.453, p<.001]$.

Aqueles que têm religião ( $M=2.86, D P=1.00)$ pontuaram significativamente mais que os que não têm religião $(M=1.74, D P=0.95)$ na dimensão transcendental. A tendência mantém-se na dimensão comunitária, com aqueles que assinalaram ter religião a obterem uma média de $3.98(D P=0.59)$ e os que assinalaram não ter a obterem uma média de 3.82 ( $D P=0.60)$. Também aqui a diferença foi estatisticamente significativa $[F(1,294)=4.932, p=.027]$.

Quanto ao BES (SWLS e PANAS), os resultados não foram significativos.

\section{DISCUSSÃO E CONCLUSÕES}

Nesta pesquisa, que envolveu adultos emergentes, uma etapa de exploração e de indefinição de vários aspetos da vida (Arnett, 2000), procurou-se compreender se o sentido de vida leva ao bem-estar. Especificamente, 


\section{SENTIDO DE VIDA, BEM-ESTAR SUBJETIVO E BEM-ESTAR ESPIRITUAL EM JOVENS PORTUGUESES E BRASILEIROS}

avaliou-se a capacidade de prever o bem-estar espiritual (BEE) e o bem-estar subjetivo (BES) a partir das dimensões do sentido de vida (SV - presença e procura).

Os resultados demonstram, em ambas as amostras, que a dimensão presença de SVé preditora dos fatores do BES (satisfação com a vida, afetos positivos e afetos negativos) e do BEE, apenas com a exceção da dimensão ambiental do BEE, que remete para as relações com a natureza, o mundo físico e biológico (cuidar e proteger) (Gouveia, Marques, \& Ribeiro, 2009). No caso dos afetos negativos, tal como esperado, a relação foi inversa, sugerindo que a presença de SV é um fator protetor. Quanto à dimensão procura de SV, apenas foram encontrados resultados significativos para a amostra portuguesa, sendo que ela é preditora dos afetos negativos, da dimensão transcendental do BEE (relação do próprio com algo para além do que é humano) e da satisfação com a vida. Neste último caso, com uma associação de sentido inverso, isto é, a procura associa-se a níveis mais baixos de satisfação. Globalmente, estes resultados corroboram estudos anteriores que destacam a importância da dimensão presença de SV para um melhor bem-estar psicológico e que, por outro lado, indicam que a dimensão procura de SV se associa a menor bem-estar (e.g., Steger, Oishi, \& Kashdan, 2009) ou não estabelece com ele relações significativas, quer diretas quer indiretas (e.g., Krok, 2015).

Foi ainda possível verificar que, nos adultos emergentes brasileiros e portugueses, a religião é relevante para a presença de SV e para a dimensão transcendental do BEE, sendo estas significativamente mais elevadas no grupo de sujeitos que dizem ter uma religião. Na amostra portuguesa, estes sujeitos apresentaram ainda resultados significativamente mais elevados na procura de SV e na dimensão comunitária do BEE. Assim, observou-se que a religião influencia o SV e, em certa medida, o BEE de brasileiros e portugueses. No entanto, em ambas as amostras, não se registou qualquer efeito significativo da religião no BES. Embora se mencionem associações positivas entre religiosidade e bem-estar, existem estudos que referem apenas correlações muito modestas entre religiosidade e satisfação com a vida ou felicidade (Diener, \& Clifton, 2002), o que se coaduna com o sentido dos resultados da presente investigação. Com amostras de estudantes universitários, tal como neste estudo, há pesquisas (Francis, Ok, \& Robbins, 2017) que apenas encontraram uma baixa associação entre religiosidade e felicidade e há mesmo quem refira uma associação negativa entre religiosidade intrínseca e BES (Machado et al., 2018). De forma a explicar as associações positivas relevantes, há autores que propõem a existência de um efeito mediador do SV entre religião e BES, sendo que a religião facilitará o SV e este terá efeito no bem-estar (Chamberlain, \& Zika, 1992; Krok, 2015). Dado que a presente pesquisa destaca a relevância da religião para 0 SV, e deste para o BES e BEE, futuramente, será interessante explorar relações de mediação ou moderação.

Numa comparação transcultural, verificaram-se resultados significativamente mais elevados a nível do SV (procura e presença) e na dimensão transcendental do BEE na amostra brasileira comparativamente à portuguesa. Este resultado está de acordo com literatura que refere que construtos como 0 sentido de vida e o bem-estar são influenciados por fatores culturais (Kleftaras, \& Psarra, 2012).

Acrescente-se que apenas foram encontradas diferenças em função do sexo na amostra brasileira, com 0 sexo masculino a revelar significativamente mais afetos positivos do que o feminino, sendo que esta tendência já foi anteriormente reportada (e.g., Morgado \& Vale Dias, 2013; Tremblay, 2010, cit. in Vale Dias et al., 2015; Vale-Dias, \& Berardo, 2019).

Em suma, embora esta investigação encerre limitações, dada a não representatividade das amostras e o facto de ter utilizado apenas questionários de autorresposta, foi possível compreender que, na relação existente entre o SV e o bem-estar (subjetivo e espiritual), se destaca sobretudo a presença de SV. É também possível sugerir 0 interesse de futuros estudos que esclareçam relações de mediação e o papel de fatores tais como a cultura ou a personalidade dos sujeitos. Finalmente, considera-se determinante 0 aprofundamento do estudo e acompanhamento dos jovens universitários, adultos emergentes, nos três pilares estudados, sentido de vida, bem-estar subjetivo e bem-estar espiritual, dadas as relações evidenciadas. A psicologia positiva, no âmbito da qual estes conceitos recebem especial atenção, surge como um contexto importante suscetível de proporcionar linhas orientadoras no acompanhamento da exploração identitária destes adultos emergentes, rumo ao equilíbrio e felicidade. 


\section{REFERÊNCIAS BIBLIOGRÁFICAS}

Albuquerque, A.S., \& Tróccoli, B. T. (2004). Desenvolvimento de uma escala de bem-estar subjetivo. Psicologia: teoria e pesquisa, 20(2), 153-164. http://www.scielo.br/pdt/ptp/v20n2/a08v20n2

Arnett, J.J. (2000). Emerging adulthood: A theory of development from late teens through the twenties. American Psychologist, 55, 469-480. https://www.ncbi.nlm.nih.gov/pubmed/10842426/

Barros, J. (2009). Busca e cura de sentido para a vida. Psychologica, 51, 93-100.

Baumeister R.F. (1991). Meanings of life. New York: Guilford.

Carvalho, M.P. \& Vale Dias, M.L. (2013). Roads to positive self-development: styles of coping that predict wellbeing. International Journal of Developmental and Educational Psychology, XXV, 1(2), 383-392. http://infad.eu/RevistalNFAD/2013/n1/volumen2/INFAD_010225_383-392.pdf

Chamberlain, K., \& Zika, S. (1992). Religiosity, meaning in life, and psychological well-being. In J. F. Schumaker (Ed.), Religion and mental health (pp. 138-148). New York: Oxford University Press.

Damásio, B., \& Koller, B.S. (2015), Meaning in Life Questionnaire: Adaptation process and psychometric properties of the Brazilian version. Revista Latinoamericana de Psicología, 47 Acedido 22/3/18: $<$ http://www.redalyc.org/articul0.0a?id=80540729005> ISSN 0120-0534

Diener, E., \& Clifton, D. (2002). Life satisfaction and religiosity in broad probability samples. Psychological Inquiry, 13(3), 206-209.

Diener, E, Lucas, R.E. \& Oishi, S. (2002). Subjective Well-Being: The Science of Happiness and Life Satisfaction. In Snyder, C. R, Lopez, S. J, Handbook of Positive Psychology. Oxford: University press.

Dohms, K., Davoglio, T. \& Lettnin, C. (2015), Validação do questionário de bem-estar espiritual: resultados preliminares para 0 contexto brasileiro, Acedido em 22/4/2020: http://uece.br/endipe2014/ebooks/livr03/469\%20VALIDAÇÃ0\%20D0\%20QUESTIONÁRI0\%20DE\%20BE MESTAR\%20ESPIRITUAL\%2ORESULTAD0S\%20PRELIMINARES\%20PARA\%200\%20CONTEXT0\%20BRA SILEIRO.pdf

Emmons, R.A. (2003). Personal goals, life meaning, and virtue: Wellsprings of a positive life. In C. Keyes \& J. Haidt (Eds.), Flourishing: Positive psychology and the well-lived life (pp. 105-128). Washington: American Psychological Association.

Francis, L.J., Ok, Ü, \& Robbins, M. (2017). Religion and happiness: A study among university students in Turkey. Journal of Religion and Health, 56(4),1335-1347. doi: 10.1007/s10943-016-0189-8

Frankl, V.E. (1965). The doctor and the soul: From psychotherapy to logotherapy. New York: Vintage Books.

Frankl, V.E. (1979). The unheard cry for meaning: Psychotherapy and humanism. New York: Simon and Schuster.

Gouveia, M.J., Marques, M., \& Pais-Ribeiro, J.L. (2009). Versão portuguesa do questionário de bem-estar espiritual (SWBQ): análise confirmatória da sua estrutura factorial. Psicologia, Saúde \& Doenças, 10(2), 285293. http://www.scielo.mec.pt/pdf/psd/v10n2/v10n2a11.pdf

Galinha, I.C., \& Pais-Ribeiro, J.L. (2005). Contribuição para 0 estudo da versão portuguesa da Positive and Negative Affect Schedule (PANAS): II-Estudo psicométrico. Análise psicológica, 23(2), 219-227.

Kleftaras, G. \& Psarra, E. (2012). Meaning in Life, Psychological Well-Being and Depressive Symptomatology: A Comparative Study. Psychology, 3, 337-345. doi: 10.4236/psych.2012.34048.

Krok, D. (2015). The Role of Meaning in Life Within the Relations of Religious Coping and Psychological WellBeing. Journal of religion and health, 54(6), 2292-2308. https://doi.org/10.1007/s10943-014-9983-3

Machado, L, de Souza, CTN, Nunes, R0, de Santana, CN, de Araujo, CF, \& Cantilino, A (2018). Subjective wellbeing, religiosity and anxiety: a cross-sectional study applied to a sample of Brazilian medical students. Trends in Psychiatry and Psychotherapy, 40(3), 185-192. http://dx.doi.org/10.1590/2237-60892017-0070

Marques, L.F., Sarriera, J.C., \& Dell'aglio, D.D. (2009). Adaptação e validação da Escala de Bem-Estar Espiritual (EBE). Avaliação Psicológica, 8(2), 179-186. Retrieved from http://pepsic.bvsalud.org/pdf/avp/v8n2/v8n2a04.pdf 
Morgado, A.M., \& Vale Dias, M.L. (2013). The antisocial phenomenon in adolescence: what is literature telling us? Aggression and Violent Behavior, 18, 436-443. doi: 10.1016/j.avb.2013.05.004

Simões, A. (1992). Ulterior validação de uma escala de satisfação com a vida (SWLS). Revista Portuguesa de Pedagogia, XXVI, 3, 503-515. https://www.scienceopen.com/document?vid=8cddf05e-d08a-4495-b28aa729aa639c57

Simões, A., Oliveira, A.L., Lima, M.P., Vieira, C.M. C., \& Nogueira, S.M. (2010). 0 MLQ: Um instrumento para avaliar 0 sentido da vida. Psicologia, Educação e Cultura, XIV, 2, 247-261. http://cic.pt/pec/default.asp? Iink=_Volume \% 20 XIV, \% 20 N $0 \% 202 \% 20$ $\% 20$ Dezembro\%202010\&Num=603\&Vol=27

Steger M.F. (2012). Making meaning in life. Psychological Inquiry, 23(4), 381-385. doi: 10.1080/1047840X.2012.720832.

Steger, M.F., Oishi, S. \& Kesebir, S. (2011) Is a Life without Meaning Satisfying? The Moderating Role of the Search for Meaning in Satisfaction with Life Judgments, Journal of Positive Psychology, 6, 173-180. DOl:10.1080/17439760.2011.569171

Steger, M.F., Oishi, S., \& Kashdan, T.B. (2009). Meaning in life across the life span: Levels and correlates of meaning in life from emerging adulthood to older adulthood. Journal of Positive Psychology, 4(1), 43-52. D0I: 10.1080/17439760802303127

Vale Dias, M.L. \& Berardo, C. (2019). Parental attitudes' perceptions and adolescents behaviour problems. International Journal of Developmental and Educational Psychology, 2(1),135-144. DOI: https://doi.org/10.17060/ijodaep.2019.n2.v2.1749

Vale Dias, M.L., Fonseca, A., Franco-Borges, G., Vaz-Rebelo, P., Oliveira, M., Moreira, A., \& De Man, J. (2015). Gender differences in strain, negative emotions, delinquency, substance use: implications for the general strain theory. Paper Presented at the 17th ECDP - September 8-12, Braga, Portugal.

Wong, P.T.P. (2000). Meaning in life and meaning in death in successful aging. In A. Tomer (Ed.), Death attitudes and older adults: Theories, concepts, and application (pp. 23-35). Philadelphia: Taylor and Francis. 